\title{
Future NTP Development Synergy Leveraged from Current J-2X Engine Development
}

\author{
Richard O. Ballard \\ Liquid Engine and Main Propulsion Systems Branch, NASA Marshall Space Flight Center, AL 35812 \\ 256-544-7015, Richard.O.Ballard@nasa.gov
}

\begin{abstract}
This paper is a discussion of how the many long-lead development elements required for the realization of a future nuclear thermal propulsion (NTP) system can be effectively leveraged from the ongoing work being conducted on the J-2X engine program for the Constellation Program. Development studies conducted to date for NTP forward planning have identified a number of technical areas that will require advancement to acceptable technology readiness levels (TRLs) before they can be utilized in NTP system development. These include high-temperature, high-area ratio nozzle extension; long-life, low-NPSP turbomachinery; and low-boiloff propellant management; and a qualified nuclear fuel element. The current J-2X program is working many of these areas that can be leveraged to support NTP development in a highly compatible and synergistic fashion. In addition to supporting technical development, there are other programmatic issues being worked in the J-2X program that can be leveraged by a future NTP development program. These include compliance with recently-evolved space system requirements such as human-rating, fault tolerance and fracture control. These and other similar mandatory system requirements have been adopted by NASA and can result in a significant technical impact beyond elevation of the root technologies required by NTP. Finally, the exploitation of experience, methodologies, and procedures developed by the J-2X program in the areas of verification, qualification, certification, altitude simulation testing, and facility definition will be especially applicable to a future NTP system. The similarities in system mission (in-space propulsion) and operational environment (vacuum, zero-gee) between J-2X and NTP make this highly synergistic. Thus, it can be shown that the collective benefit of leveraging experience and technologies developed during the J-2X program can result in significant savings in development cost and schedule for NTP.
\end{abstract}

Keywords: nuclear thermal propulsion, NTP, lessons learned, fundamental root cause, risk mitigation.

PACS: $28.50 . \mathrm{Ky}$; Propulsion reactors.

\section{INTRODUCTION}

The Constellation Program (CxP) was formed as a result of the release of the President's Vision for Space Exploration (VSE) in 2004. During the initial formulation stages of the CxP, a number of unpublished NASAinternal architecture road-mapping studies were conducted to identify the optimal development path to enable manned exploration of the moon and Mars defined in the VSE. The final road-mapping study, the Exploration Systems Architecture Study (ESAS) (NASA, 2005a), was publicly released and defined the path that NASA will pursue in achieving the VSE objectives. The ESAS led to the initiation of the Ares-I Crew Launch Vehicle (CLV) and Ares-V Cargo Launch Vehicle (CaLV) development programs with the goals of permitting the decommissioning of the Space Transportation System (STS) while continuing access to the International Space Station (ISS), as well as enabling the achievement of the VSE. In order to manage cost and schedule for system development, both Ares launch vehicles utilize common elements whenever possible, such as the solid rocket boosters developed for the STS, and the J-2X upper stage engine derived from the J-2 engine used on the Saturn-IB and Saturn-V launch vehicles on the Apollo program. The J-2X engine will be used both on the Ares-I upper stage and the Ares-V Earth Departure Stage (EDS).

In addition to the ESAS road-mapping effort, other similar planning activities have been conducted to identify gaps in critical technologies needed to achieve crewed return to the moon and eventual pursuit of crewed exploration of Mars. These Design Reference Mission (DRM) studies for Mars exploration have identified nuclear thermal propulsion (NTP) as the leading propulsion option because of its high specific impulse $\left(\mathrm{I}_{\mathrm{sp}} \sim 875-1000\right.$ seconds) and 
an acceptable engine thrust-to-weight ratio ( 3-10). The NTP concept was demonstrated to a Technology Readiness Level (TRL) approaching 6 (i.e., system level testing in a relevant environment) during the Rover/NERVA NTP programs conducted $\sim 1955-1972$. However, since the termination of the Rover/NERVA program, the technology base and supporting infrastructure has atrophied such that significant technology gaps have developed that must be bridged before a human-rated NTP system can be made available to support the full realization of the VSE.

Another road-mapping activity conducted during the initial phase of the Constellation program was the Capabilities, Requirements, Analysis and Integration (CRAI) study, an internal NASA study which was to evaluate the critical technologies needed to support executing the VSE and to identify technology gaps that required development investment to close. A large number of technology areas were evaluated for possible investment, including NTP. The results of the CRAI study showed that the successful development of a NTP system for a Mars exploration mission had a number of key technologies that required development in order to enable system development at an acceptable technical risk. These included, but are not limited to:

- Large area ratio nozzle extension, probably radiatively cooled, integrated with a regeneratively-cooled thrust chamber/nozzle section, compatible with hot hydrogen exposure for extended operation.

- Highly reliable liquid hydrogen (LH2) turbomachinery capable of extended operation ( 2 hours total) under a wide range of propellant inlet conditions, including low net positive suction pressure (NPSP).

NTP development planning studies conducted internally at NASA show an extended development period on the order of $\sim 12$ years or more in order to mature and certify a human-rated NTP system that will enable crewed exploration missions beyond cislunar space. A development cycle for a comparable conventional liquid propellant rocket engine generally requires 8-10 years, but a NTP development effort would require more time to accomplish fuel development, permitting, and recovery/build-up of necessary infrastructure. The NTP planning study detailed a notional NTP development effort including build-up of ground infrastructure, system development and integration, extensive testing and technology risk mitigation. After the study was concluded, it was identified that there are areas in the current scope of the ongoing J-2X engine development program that are concurrent with the identified needs of a notional NTP development program.

The focus of this paper is to highlight areas of technical and programmatic activity being pursued by the ongoing $\mathrm{J}-2 \mathrm{X}$ program that can be effectively leveraged by a notional NTP development program to enable the Mars exploration mission. Many of the accomplishments of the J-2X program described in this paper can be readily applied toward a follow-on nuclear in-space propulsion system and the synergy provided will help reduce time and resources needed while assuring a safe, reliable propulsion system to enable exploration missions beyond cislunar space.

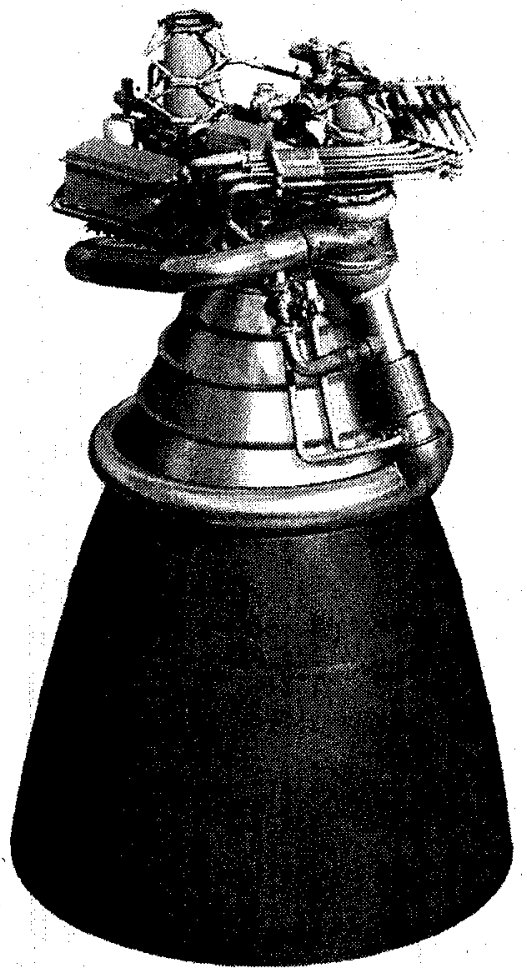

FIGURE 1. $J$-2X Engine.

\section{THE J-2X PROGRAM}

The J-2X Upper Stage Engine/Earth Departure Stage Engine (USE/EDSE) is a LH2, liquid oxygen (LO2) rocket engine based upon the heritage of the J-2 rocket engine used during the Apollo era (see Figure 1). Its mission will be to provide propulsion for the Ares-I Upper Stage and the Ares-V EDS. Ares-V mission requirements for the J2X engine include the following: start at altitude (i.e., vacuum), operate at either of two power levels, shutdown for disposal or loiter on orbit for up to 100 days, restart on command, operate with sufficient impulse for earth departure and shutdown: The J-2X engine requirements for the Ares-1 mission are a subset of those identified for the Ares-V mission. The J-2X operates on a gas generator power cycle that produces $294,000 \mathrm{lbf}$ of thrust at a nominal specific impulse of 448 seconds in vacuum and can be operated at two thrust levels called "primary" and "secondary" mode. 
In light of the fact that the goal of the J-2X engine program will be to develop the first human-rated rocket engine in the United States in over 30 years (the last was the Space Shuttle Main Engine, SSME), it should be understood that the program will be able to draw upon a generation of technology and operational experience that has evolved since the SSME was developed for the Space Shuttle. In addition, the J-2X program will be guided by a selection of standards, policies and guidelines that have been baselined and mandated for implementation under the NASA programs in general and the Constellation program in particular. Accomplishing the compliance of the J-2X program with these technical and programmatic mandates will be a challenge that any following propulsion programs will also need to achieve, including NTP.

\section{NTP TECHNOLOGY RISK REDUCTION SYNERGY}

The benefits from the current J-2X effort that can be exploited by a notional NTP development program can be derived from many sources, such as:

- Results of system- and vehicle-level trades. Any system development program has to navigate a development path that is encumbered with numerous problematic issues that have to be addressed by trade studies to converge on an optimal solution for the propulsion system and the vehicle that it powers. Most of the major design decisions used to guide J-2X development have been documented and will certainly be of use toward NTP development as well. The role of in-space propulsion system mandates numerous unique requirements in terms of function and environment, which will be compatible between both systems.

- Material properties characterization. The Rover/NERVA effort produced a significant technology base of materials properties data that is still largely relevant today. However, the selection of materials available for propulsion system development has changed considerably since the last significant effort was conducted on NTP development. The material properties data generated by $J-2 X$ will be a timely addition to the Rover/NERVA materials database, but would need to be extended by radioactive exposure response data in order to be directly applicable toward NTP design.

- Advancement in the institutional experience level of personnel supporting system development. As previously stated, the last human-rated rocket propulsion system developed by NASA was the SSME for the Space Shuttle. Since then, several rocket development programs have been initiated (STME, RS-83, RS-84, TR107, COBRA, Fastrac), but were cancelled before the development cycle was completed. At the conclusion of the J-2X design, development, test and evaluation (DDT\&E) cycle, the personnel supporting the program will have gained valuable experience in what is required to accomplish a similar effort for a comparable NTP DDT\&E effort.

- Development planning and implementation. The broad scope of the J-2X program extends beyond that of simply development of the engine system itself. Because of the in-space conditions that the engine will operate in, specialized ground test facilities are being constructed to support system testing under mission environments. The coordination skills and processes used to efficiently orchestrate the development of the flight system with the supporting infrastructure is especially applicable toward a notional NTP program.

- Lessons Learned from activities involving development, test, facilities, vehicle integration, mission operations, program management, and systems engineering and integration (SE\&I), to name a few.

In addition to the above opportunities for leveraging the experience gained during the $\mathrm{J}-2 \mathrm{X}$ program, there are also several key areas of technical risk that a notional NTP program can benefit from as a result of the work being conducted to support J-2X engine development. Significant time and resources are being expended by the J-2X program to mitigate these risk areas, and the aggregate results can be leveraged by any subsequent rocket propulsion development efforts, especially those developed to support planetary exploration missions beyond Low Earth Orbit (LEO), a mission ideally suited for a NTP system. Some descriptions of these areas are discussed in the following sections. 


\section{Low NPSP LH2 Turbomachinery}

As a consequence of the zero-gravity environment in which they operate, space vehicle upper stages using liquid propellants often have to contend with the variable quality of the propellants, which exhibit indefinite separation between the liquid phase propellant and the gaseous phase ullage. This can generally be addressed by subjecting the stage to a low propulsive impulse, usually either through propellant-settling rocket thrusters or operating the main propulsion system at low power (also known as idle mode), to settle the propellants at the bottom of the tank and assure liquid phase flow at the inlet of the rocket engine system. In addition, this operational problem is compounded by limits on the maximum operating pressure of the propellant tank that can collectively degrade the quality of propellants at the engine inlet to the point where the low NPSP can result in an elevated risk of cavitation in the turbopump inducer that will affect system performance and potentially result in significant, possibly catastrophic, engine damage. In order to enable a higher operating pressure in the propellant tank, the tank wall thicknesses would need to be higher, which would cause a rapidly escalating weight impact to the vehicle. Trade studies conducted between the engine and the vehicle would need to focus on convergence toward an optimal balance between the propellant tank weight as a consequence of its operating pressure and the minimum propellant inlet pressure that the engine can safely operate with.

The J-2X engine is being designed to operate in a mission profile that can subject it to low NPSP conditions at the engine inlet. This would be especially the case for $\mathrm{J}-2 \mathrm{X}$ missions on the Ares-V EDS, where it is required to execute a trans-lunar injection (TLI) burn up to 100 days after being launched to orbit. The Mark-29F LH2 turbopump design being developed for the J-2X fuel turbopump (FTP) is well understood, having been used previously on the J-2S and XRS-2200 engine programs.

It is understood that the mission profile a notional NTP system would need to accomplish requires multiple longduration engine operations over an extended mission phase. This means that the turbopump used by a NTP system would need to be able to operate reliably, safely and for much longer durations than the current Mark-29F is being designed for. However, much of this can be accomplished by structural reinforcement and selected redesign of Mark-29F elements. Further, the experience gained during the development and flight operation of the Mark-29F FTP could be leveraged to provide a solid foundation for a derived design that added emphasis on high reliability and extended duration in addition to low NPSP operation.

It should be emphasized that neither rocket engines or their constituent components should be considered as "plugand-play" items to be swapped out freely between vehicles or other rocket engine systems. A significant effort is involved in characterizing the major operational characteristics of a component and rigorously integrating it into the aggregate engine system to assure that the system will be able to perform its intended mission with an acceptable operational margin. However, for the case of a NTP development effort, it is feasible to leverage the experience and technology generated for a specific design, such as in the J-2X engine, to serve as a point of departure for a derived NTP design.

\section{High Area Ratio Nozzle Extension}

In order to optimize the system performance (e.g., specific impulse) of a NTP system, it is necessary to derive the maximum benefit from the system operating temperature and pressure, and the nozzle exit ratio. The operating conditions of the NTP system will be traded against the system requirements and the available fuel technology base, but the expansion ratio of the nozzle will be constrained by the material properties that it is fabricated from and the physical size and weight constraints that will allow it to be place in orbit. For in-space rocket propulsion systems, an effective means of maximizing specific impulse is to increase the nozzle area ratio as high as the weight and volume limitations of the ground launch architecture will support.

As an upper stage engine, the J-2X will have the highest specific impulse of any gas generator cycle engine in its thrust class (448 seconds vacuum $I_{\text {sp }}$ ). This is achieved by high chamber pressure and area ratio. The 92:1 area ratio is achieved by the utilization of a large radiatively-cooled nozzle extension mounted after the regeneratively-cooled nozzle. The large size and high heat load of the nozzle extension requires that it be made of a robust hightemperature material such as refractory metal alloy or carbon-carbon composite. At this time, the current design baseline for the $\mathrm{J}-2 \mathrm{X}$ nozzle extension is a composite material design, but its unprecedented size requires significant technical risk to be overcome. Although composite nozzle extensions have been used in current rocket engine systems (i.e., RL10B-2), the nozzle extension being developed for the J-2X will be by far the largest. 
It is likely that any NTP system developed in the future will also require a high area ratio to enable a high specific impulse, which will permit the leveraging of much of the results of the nozzle extension development currently pursued by the $\mathrm{J}-2 \mathrm{X}$ program. This will be especially relevant in the disciplines of structural and thermal analyses, as well as fracture control applied to a large composite structure. The experience gained in system testing a large nozzle extension under altitude conditions may also provide valuable insight into the design and operation of a NTP system test facility, if needed. The achievements of the J-2X nozzle extension development will not, however, be able to take into account the radiation loads that an operational NTP engine will subject the nozzle extension to, but it will provide an excellent point of departure for additional development.

Another of the concerns associated with developing a rocket engine with a high area ratio nozzle is being able to test it on the ground. Because of the backpressure from atmospheric pressure, nozzles with area ratios generally higher than 25:1 are prohibited from being operated at ground conditions because the under-expanded exhaust in the nozzle can generate unstable sideloads against the nozzle wall that can result in catastrophic engine damage. Being able to operate an in-space rocket system on the ground requires that it be mounted in a vacuum test cell that can simulate the ambient pressure conditions at the system's operational altitude (i.e., near-vacuum). In addition, the long orbital loiter time (up to 100 days) required by the J-2X system for Ares-V missions will also necessitate that the test cell be fitted with cryo-panels to enable the cell to cold-soak the system to simulate temperature conditions in orbit. The design and operational experience gained from J-2X altitude testing would be of direct benefit to a follow-on NTP program, as well as the results and Lessons Learned from the testing itself.

\section{NTP SYSTEMS ENGINEERING SYNERGY}

System development for NASA space flight systems, especially for human-rated systems, is guided by a number of safety and design standards. Compliance with these standards is generally mandatory unless a defensible rationale can be provided for any deviation from them. Table 1 shows a listing of mandatory technical standard that are applicable for all NASA human-rated and robotic missions, although some may be exempted from rocket systems. For a nuclear systems, the list of mandatory standards is similarly long as well.

The safety standards applied to non-nuclear rocket engines originate from a variety of sources and topic areas, including industrial safety, launch range safety, military standards, NASA standards, and, if required, human rating requirements. All these standards are collective and complementary, addressing a different facet of the manufacture, handling, test, and operation of the engine system. These standards address the safety concerns presented by the rocket engine as it applies to the public and those directly involved with it. It is generally important that consideration of the safety standards be applied as early as possible to the engine design process to prevent an expensive and time-consuming corrective implementation of the necessary standard later in the engine life cycle.

TABLE 1. Mandatory Technical Standards for NASA Human-Rated and Robotic Missions.

\begin{tabular}{|c|c|c|}
\hline Discipline & Standard & Title \\
\hline Electrical & NASA-STD-4003 & Electrical Bonding For NASA Launch Vehicles, Spacecraft, Payloads, And Flight Equipment \\
\hline Electrical & NASA-STD-4005 & Low Earth Orbit Spacecraft Charging Design Standard \\
\hline Electrical & AIAA S $-111-2005$ & Qualification and Quality Requirements for Space Solar Cells \\
\hline Electrical & AIAAS-112-2005 & Qualification and Quality Requirements for Space Solar Panels \\
\hline Electrical & MIL-STD-461 & $\begin{array}{l}\text { Requirements for the Control of Electromagnetic Interference Characteristics of Subsystems } \\
\text { and Equipment }\end{array}$ \\
\hline Human Factors & NASA-STD-3000, Vol. I, II & Man-Systems Integration Standards \\
\hline Materials \& Processes & NASA-STD-5006 & General Fusion Welding Requirements For Aerospace Materials Used In Flight Hardware \\
\hline Materials \& Processes & NASA-STD-6001 & $\begin{array}{l}\text { Flammability, Odor, Off gassing And Compatibility Requirements \& Test Procedures for } \\
\text { Materials In Environments that Support Combustion (Superseding NHB-8060.1C) }\end{array}$ \\
\hline Materials \& Processes & NAS410 & National Aerospace Standard Certification and Qualification of Nondestructive Test Personnel \\
\hline Structures / MEQ & NASA-STD-5001 & Structural Design and Test Factors of Safety for Spaceflight Hardware \\
\hline Structures / MEQ & NASA-STD-5002 & Load Analyses Of Spacecraft And Payloads \\
\hline Structures / MEQ & NASA-STD-5017 & Design and Development Requirements for Mechanisms \\
\hline Structures / MEQ & AIAA S-080-1998 & Space Systems; Metallic Pressure Vessels, Pressurized Structures, and Pressure Components \\
\hline Structures / MEQ & AIAA S-081A-2006 & Standard for Space Systems - Composite Overwrapped Pressure Vessels (COPVs) \\
\hline Operations & AFSPCMAN 91.710 & Range Safely User Requirements Manual \\
\hline Program Control & NASA-STD-6002 & Applying Data Matrix Identification Symbols on Aerospace Parts \\
\hline Test \& Verification & NASA-STD-7001 & Payload Vibroacoustic Test Criteria \\
\hline Test \& Verification & NASA-STD-7002 & Payload Test Requirements \\
\hline Test \& Verification & NASA-STD-7003 & Pyroshock Test Criteria \\
\hline
\end{tabular}


The design of non-nuclear rocket engines is governed by specific standards, originating either from the government or the engine contractor. In instances where the content of the contractor standard overlaps that of a government standard, it must show that it "meets or exceeds" the requirements specified in the government standard. These standards define the minimum criteria that must be met in the system design and are the result of exhaustive experience in the topic areas. Examples of the government standards used include structural margins of safety, fracture control, non-destructive evaluation and materials selection.

It is important to note that the implementation of these standards and requirements is by no means an easy exercise. The effort that the $\mathrm{J}-2 \mathrm{X}$ program has expended in addressing these mandates is significant and will need to be similarly attended to by any subsequent propulsion system development effort, such as NTP.

\section{Human-Rating Requirements}

NASA's policy is to protect the health and safety of humans involved in or exposed to space activities, specifically the public, crew, passengers, and ground personnel (NASA, 2005b). The requirements for human-rating a propulsion system has been captured in NASA NPR 8705.2, which is applicable to all space systems involving humans or interfacing with human space systems prior to becoming operational and throughout the system's use. A program is eligible for human-rating certification only if it meets engineering requirements, health requirements, and safety requirements. These requirements are being implemented in the $\mathrm{J}-2 \mathrm{X}$ program and would also be applicable to NTP.

\section{Structural Margins of Safety}

NASA mandates minimum structural factors of safety in most of the flight systems used in crewed and robotic exploration missions. These structural standards address strength and life assessment requirements, including tests and analyses to structurally qualify rocket engines, as well as the minimum structural factors of safety acceptable to NASA (NASA, 2006a). These requirements are captured in NASA-STD-5012, and specify analyses and test factors, margins, and other parameters where appropriate.

The J-2X program has defined the structural requirements for the engine in accordance with the NASA-STD-5012 standard to ensure a structurally sound design that will assist the program in meeting engine performance goals. The implementation of NASA-STD-5012 by the J-2X program provides many Lessons Learned that can be leveraged by a notional NTP development effort.

\section{Fracture Control}

NASA's policy is to produce flight systems with a high degree of reliability and safety. This is accomplished through good engineering practices in the design, analyses, inspections, testing, fabrication, and operation of flight structures. In keeping with this policy, all human-rated space systems shall be subjected to fracture control to preclude catastrophic failure. The requirements associated with fracture control have been levied on all flight systems contained in the Constellation program (NASA, 2006b).

Fracture control encompasses the consequences of naturally occurring and service-induced flaws, damage, or cracks into a part or structure. In keeping with the mandates of NASA-STD-5019, the J-2X program has established the fracture control plan that will mitigate the risk associated with fracture-induced system failure. The implementation of a viable fracture control program for a rocket propulsion system was first accomplished by the J-2X and can be of use to other rocket system development efforts, such as NTP.

\section{CONCLUSION}

It is clear that a number of similarities exist between the ongoing $\mathrm{J}-2 \mathrm{X}$ rocket engine program and projected needs of a notional NTP development effort. The achievements and experience gained by the J-2X program can be of direct benefit toward NTP, and can be identified and applied in a timely manner in order to generate the maximum synergy between the two propulsion development efforts. 


\section{REFERENCES}

NASA, "NASA's Exploration Systems Architecture Study," Final Report, NASA, November 2005 (a).

NASA, "Human-Rating Requirements for Space Systems," NASA Procedural Requirements NPR 8705.2A, 7 February 2005 (b).

NASA, "Strength and Life Assessment Requirements for Liquid Fueled Space Propulsion System Engines," NASA-STD-5012, 13 June 2006 (a).

NASA, "Fracture Control Requirements for Spaceflight Hardware," Interim Standard, NASA-STD-(I)-5019, 12 September 2006 (b). 